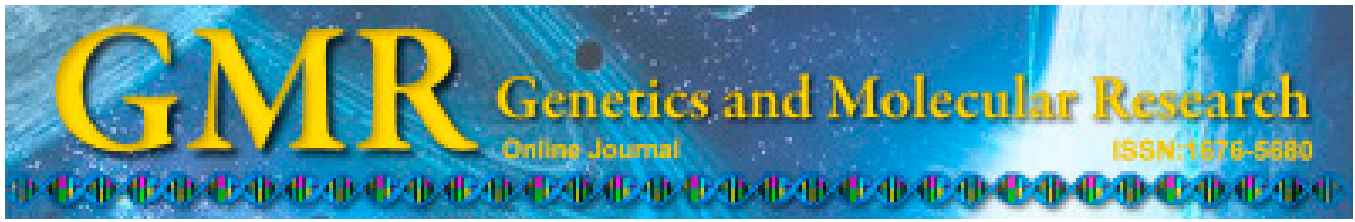

\title{
Qualitative analysis of the vaginal microbiota of healthy cattle and cattle with genital-tract disease
}

\author{
N.F. Rodrigues ${ }^{1}$, J. Kästle ${ }^{1}$, T.J.D. Coutinho ${ }^{1}$, A.T. Amorim ${ }^{2,3}$, G.B. Campos ${ }^{2,3}$, \\ V.M. Santos ${ }^{2,3}$, L.M. Marques ${ }^{2,3}$, J. Timenetsky ${ }^{2}$ and S.T. de Farias ${ }^{1}$ \\ ${ }^{1}$ Departamento de Biologia Molecular, Centro de Ciências Exatas e da Natureza, \\ Universidade Federal da Paraíba, João Pessoa, PB, Brasil \\ ${ }^{2}$ Departamento de Microbiologia, Instituto de Ciências Biomédicas, \\ Universidade de São Paulo, São Paulo, SP, Brasil \\ ${ }^{3}$ Núcleo de Tecnologia em Saúde, Instituto Multidisciplinar em Saúde, \\ Campus Anísio Teixeira, Universidade Federal da Bahia, \\ Vitória da Conquista, BA, Brasil \\ Corresponding authors: N.F. Rodrigues / S.T. de Farias \\ E-mail: nureyevrod@gmail.com / stfarias@yahoo.com.br
}

Genet. Mol. Res. 14 (2): 6518-6528 (2015)

Received June 27, 2014

Accepted November 21, 2014

Published June 12, 2015

DOI http://dx.doi.org/10.4238/2015.June.12.4

\begin{abstract}
The microbial community of the reproductive apparatus, when known, can provide information about the health of the host. Metagenomics has been used to characterize and obtain genetic information about microbial communities in various environments and can relate certain diseases with changes in this community composition. In this study, samples of vaginal surface mucosal secretions were collected from five healthy cows and five cows that showed symptoms of reproductive disorders. Following high-throughput sequencing of the isolated microbial DNA, data were processed using the Mothur software to remove low-quality sequences and chimeras, and released to the Ribosomal Database Project for classification of operational taxonomic units (OTUs). Local BLASTn was performed and results were
\end{abstract}


loaded into the MEGAN program for viewing profiles and taxonomic microbial attributes. The control profile comprised a total of 15 taxa, with Bacteroides, Enterobacteriaceae, and Victivallis comprising the highest representation of OTUs; the reproductive disorder-positive profile comprised 68 taxa, with Bacteroides, Enterobacteriaceae, Histophilus, Victivallis, Alistipes, and Coriobacteriaceae being the taxa with the most OTU representation. A change was observed in both the community composition as well as in the microbial attributes of the profiles, suggesting that a relationship might exist between the pathogen and representative taxa, reflecting the production of metabolites to disease progression.

Key words: Metagenomics; Reproductive disorders; 16S rRNA

\section{INTRODUCTION}

Cattle are affected by several diseases related to the genital tract caused by various pathogens, such as bacteria of the genera Bacteroides, Mycoplasma, Histophilus, Fusobacterium, and Prevotella; and the species Escherichia coli and Streptococcus spp, among others (Pfützner and Sachse, 1996; Corbeil, 2007; LeBlanc, 2008). These diseases have common aspects, but sometimes the symptoms are distinct. In some cases, the diagnosis of infection can be based in these exclusive symptoms, which correspond to specific informations to the evaluated pathogen, that provide additional information that can be initially considered for choose tests to confirm the diagnosis; this is the most common method for deciding on the course of treatment for control of the disease, initially in a given animal, can be extended to a larger scale. Recent studies that incorporate disease assessment in a clinical setting have been notable for their focus on ecological interactions, based on the new data provided through a metagenomic approach. Studies on the composition of microbial communities in diverse environments have revealed the existence of intricate ecological interactions (Kent and Triplett, 2002; Baudoin et al., 2003), and how environmental degradation and other anthropogenic activities impact these communities (Garbeva et al., 2004; Lynch et al., 2004). In hosts, studies have shown that changes in the constitution of communities can lead to health disorders (Seksik et al., 2003; Ott et al., 2004; Turnbaugh and Gordon, 2009); however, the identification of the microbial community requires cultivation of isolated strains in vitro, thus excluding several organisms whose unique physiologies do not allow cultivation. A new era in microbial ecology was initiated with the sequencing of ribosomal RNA (rRNA) genes by metagenomics, which allowed the description of unculturable bacteria (Singh et al., 2009). Metagenomics has permitted the collection and analysis of biological information without the need for culture of the organisms in the sample. The "Microbiome" project, a term analogous to "biome" in ecology and referring to the microbial community of a particular environment (e.g., a specific location in the body of an individual), has contributed to the identification of microorganisms responsible for the balance of the health of the host (Turnbaugh and Gordon, 2009; Brown et al., 2011). The aim of this study was to characterize the microbiota of cows with reproductive disorder symptoms, and determine the relationship to components of the microbial infection in comparison with a normal microbiota. 


\section{MATERIAL AND METHODS}

\section{Sample collection and DNA isolation and purification}

Samples were collected from five female bovids which showed clinical signs of reproductive disorders (whitish vagina, purulent vulvar discharge, and inflamed hyperemic vulvar mucosa with granulomatous vulvovaginitis) (Nicholas et al., 2008), and five which showed no aspects of the disorders. After disinfection of the vaginal external surface of the animal, a sample from the site was obtained via a vulvaginal swab, with care taken to avoid the vestibule and clitoral fossa of the animal. After insertion into the vestibule, the swab was initially directed almost completely dorsally before redirecting cranially, and then rolled over the cranial vaginal mucosa. The swabs were placed in $25 \mathrm{~mL}$ transport medium and stored at $-20^{\circ} \mathrm{C}$ until further use. Bacterial genomic DNA was isolated and purified with the Invisorb ${ }^{\circledR}$ Spin Blood Midi Kit (Stratec Molecular, Berlin, Germany). The protocol followed the manufacturer instructions, with minor modifications; i.e., a pretreatment step with the Buffer EL was not performed. After isolation, the purified DNA was eluted in $200 \mathrm{~mL}$ elution buffer. Quality and purity of the isolated genomic DNA was confirmed by agarose gel electrophoresis and spectrophotometric analysis using a NanoDrop 2000 (Thermo Fisher Scientific, Schwerte, Germany).

\section{$16 \mathrm{~S}$ primers and amplicon library generation}

Polymerase chain reaction (PCR) amplification of the16S rRNA hypervariable V5-V6 region was performed using degenerate forward (V5F-784: 5'-AAC RGG ATT AGA TAC CC3') and reverse primers (V6R-1064: 5'-CGA CRR CCA TGC ANC ACC T-3') for bacteria. The 5 '-ends of the forward primers were fused to the adapter-A followed by the key sequence; the reverse primers were fused with a truncated sequence of the adapter-Pi (TRP1). The primers were diluted in molecular biology-grade water at equimolar amounts. To prepare the amplicon library, $4 \mathrm{ng}$ metagenomic DNA from each sample, 1 U Platinum High-Fidelity Taq DNA polymerase, $5 \mathrm{mM}$ dNTPs, $2 \mathrm{mM} \mathrm{MgCl}$ (Life Technologies, Carlsbad, CA, USA), and 10 pmol premixed primers were combined in a $25-\mu \mathrm{L}$ total volume reaction mix. For PCR, the amplification conditions were $94^{\circ} \mathrm{C}$ for $3 \mathrm{~min}$, followed by 30 cycles of $94^{\circ} \mathrm{C}$ for $15 \mathrm{~s}, 55^{\circ} \mathrm{C}$ for $15 \mathrm{~s}$, and $68^{\circ} \mathrm{C}$ for $10 \mathrm{~s}$, with a final elongation step at $68^{\circ} \mathrm{C}$ for $30 \mathrm{~s}$. The PCR products were purified on a $1.5 \%$ agarose gel.

\section{Emulsion PCR and sequencing}

Emulsion PCR was performed using the Express Ion Template Kit V2.0 (Life Technologies) according to the manufacturer protocol. Sequencing of amplicon libraries was performed using the high-throughput sequencing platform: Ion Torrent Personal Genome Machine - PGM, using Sequencing kit 200 (Life Technologies) reagents following the manufacturer protocol (Part No. 4471999 Rev. B, 13. Oct. 2011) with modifications as follows: 1) the chip was washed one additional time with isopropanol and after the annealing buffer, and checked and calibrated to remove possible air bubbles resulting from these procedures; 2) the beads were loaded twice onto the 314 chip, with each loading followed by four cycles of centrifugation at maximum speed for $15 \mathrm{~s}$ (Mini Star, VWR International GmbH, Darmstadt, Germany) and stirring at $3000 \mathrm{rpm}$ for $10 \mathrm{~s}$ in an IKA orbital shaker (IKA-Werke GmbH \& Co. 
KG, Staufen, Germany) equipped with a special adapter for the chip, followed by centrifugation at the end of each load for $15 \mathrm{~s}$; and 3) the entire sample was loaded onto the chip.

\section{Sequence analysis}

Raw sequencing reads were checked according to several quality criteria using the Mothur program (Schloss et al., 2009). Low-quality reads were removed as follows: 1) reads not matching the PCR primers with at most two errors; 2) sequences containing ambiguously called bases $(\mathrm{N})$; and 3) sequences with a length less than $150 \mathrm{bp}$. The sequences were screened for artificial chimeric formations using the UCHIME algorithm in Mothur. Subsequently, operational taxonomic unit (OTU) analysis was conducted on a clustering basis for each sample individually in the Ribosomal Database Project (RDP) (Cole et al., 2009). Local BLASTn was used to assign 16S rRNA gene sequences in each sample, using the database 16SMicrobial (version 02/16/2013). The output BLASTn text file was loaded into MEGAN (Huson and Mitra, 2012), and the data were used to create graphs of the profiles' comparative and microbial taxonomic tree attributes.

\section{RESULTS}

A total of 228,842 sequence reads were generated in the sequencing process; after the initial pipeline of RDP and Mothur processing, a total of 32,185 high-quality sequences were obtained. The majority of $16 \mathrm{~S}$ rRNA amplicons sequenced belonged to the hypervariable V5V6 region. Chimeric sequences were found in very small quantities (less than $1 \%$ per profile). Clustering of reads defined by a $\leq 3 \%$ sequence difference at the species level generated 594 and 1057 clusters for the control (without reproductive disorders) and positive (with disease symptoms) profiles, respectively (Table 1).

Table 1. Operational taxonomic unit counts (clusters) in positive and control profiles.

\begin{tabular}{lcrr}
\hline Profile & Distance* & N** & Clusters \\
\hline Positive & 0.03 & 31,229 & 10,754 \\
Positive & 0.05 & 31,229 & 6645 \\
Positive & 0.10 & 31,229 & 2246 \\
Control & 0.03 & 956 & 594 \\
Control & 0.05 & 956 & 442 \\
Control & 0.10 & 956 & 207 \\
\hline
\end{tabular}

*Levels distance. **Number of sequences.

Rarefaction curves showed that at the level of family $(0.10)$, we obtained a satisfactory coverage of OTUs analyzed; yet, at the species $(0.03)$ and genus $(0.05)$ levels, the asymptotes were not reached. These curves were therefore not optimal, but were still of considerable quality (Figures 1 and 2). Approximately $3.19 \%$ of the total 15,310 OTUs identified were common between profiles under investigation in this study (Table 2).

The taxonomic classification of OTUs revealed the constitution of the communities: the profile of the control animals presented OTUs corresponding to 15 taxa: Bacteroides (51 reads; 28.3\%), Enterobacteriaceae (32 reads; 17.8\%), Victivallis (13 reads; 7.2\%), Streptococcus (11 reads; 6.1\%), Selenomonadales (10 reads; 5.6\%), Treponema (10 reads; 5.6\%), Porphyromonadaceae ( 9 reads; $5 \%$ ), Alistipes ( 7 reads; 3.9\%), Coriobacteriaceae (6 reads; 3.3\%), 
Clostridium (6 reads; 3.3\%), Betaproteobacteria (5 reads; $2.8 \%$ ), Corynebacterineae (5 reads; $2.8 \%$ ), Cytophagaceae (5 reads; $2.8 \%$ ), Oscillibacter ( 5 reads; $2.8 \%$ ), and Planctomycetaceae (5 reads; $2.8 \%$ ) (Figure 3 ). The profile of animals with reproductive disorders (positive) presented OTUs corresponding to 68 taxa, and the predominant taxa were: Bacteroides (2246 reads; 35.83\%), Enterobacteriaceae (1167 reads; 18.62\%), Histophilus (551 reads; 8.79\%), Alistipes (272 reads; 4.34\%), Flavobacteriaceae (111 reads; 1.77\%), Victivallis (532 reads; $8.49 \%$ ), Coriobacteriaceae (153 reads; 2.44\%), Streptococcus (131 reads; 2.09\%), Barnesiella (127 reads; 2.03\%), and Oscillibacter (78 reads; 1.24\%) (Figure 4).

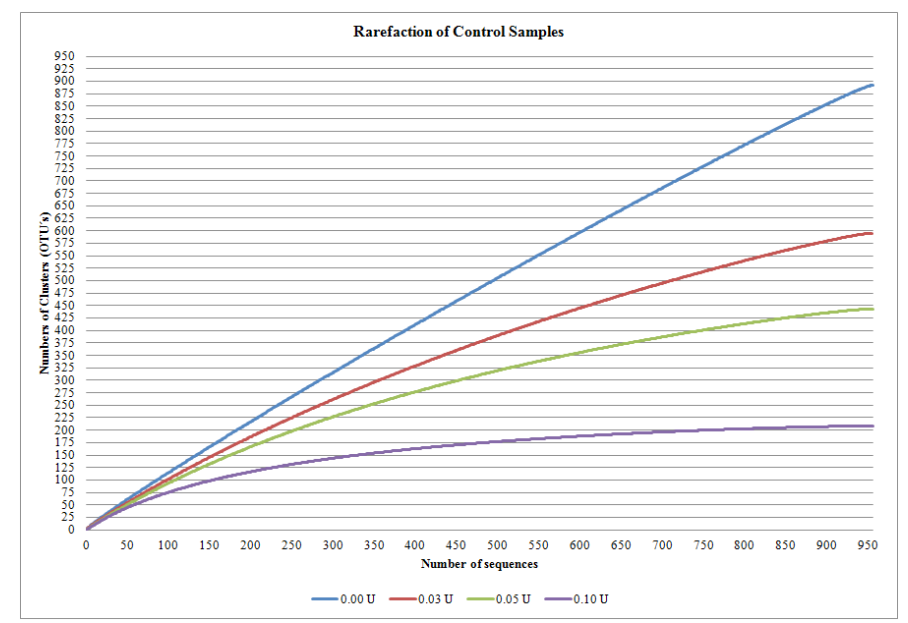

Figure 1. Curves of rarefaction of the control profiles of operational taxonomic units (OTUs) obtained from the Ribosomal Database Project (RDP) to three levels distance (90, 95, and 97\%).

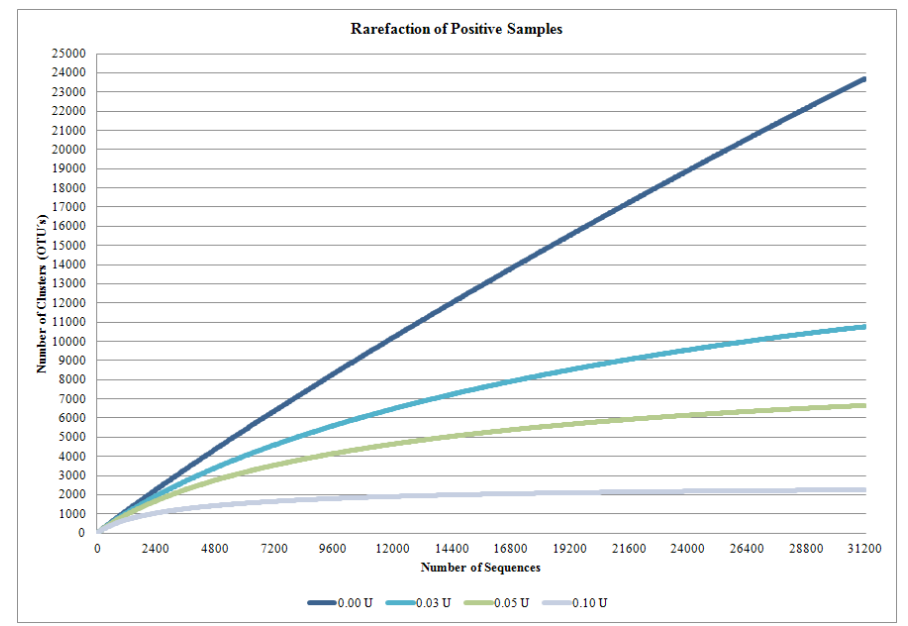

Figure 2. Curves of rarefaction of the positive profiles of operational taxonomic units (OTUs) obtained from the Ribosomal Database Project (RDP) to three levels distance (90, 95, and 97\%). 
Table 2. Operational taxonomic units (OTUs) shared between positive and control profiles.

\begin{tabular}{lcr}
\hline Distance* & Shared OTUs & \% of total OTUs \\
\hline 0.03 & 489 & 3.19 \\
0.05 & 388 & 4.49 \\
0.10 & 217 & 6.78 \\
\hline
\end{tabular}

*Levels distance.

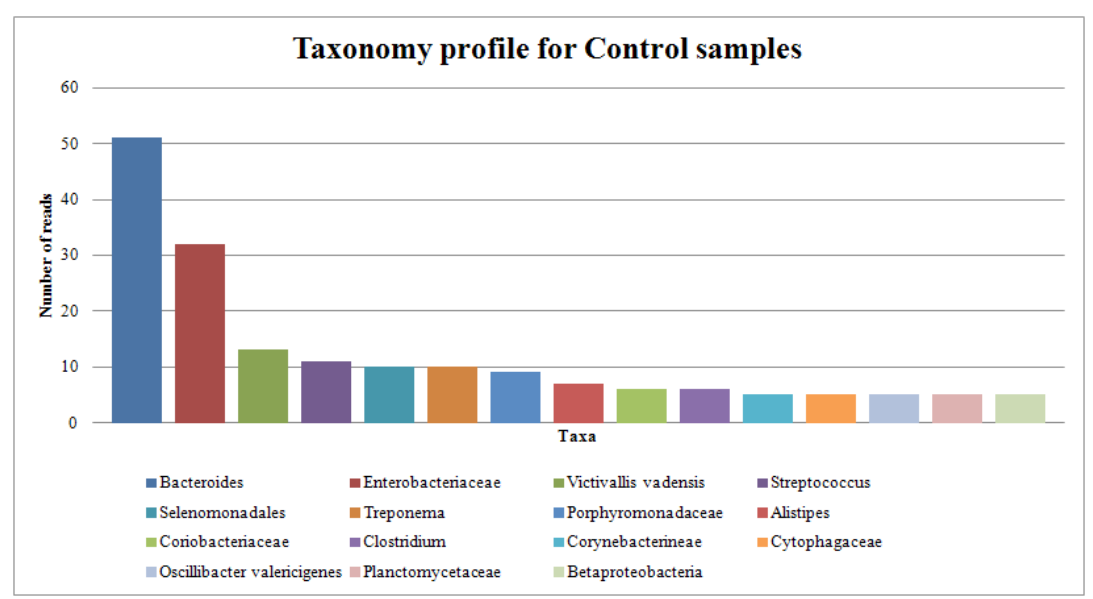

Figure 3. Taxonomic groups in the control profile, generated in MEGAN, through local BLASTn, with 95\% similarity. The taxa in legend are presented in descending order, from left to right.

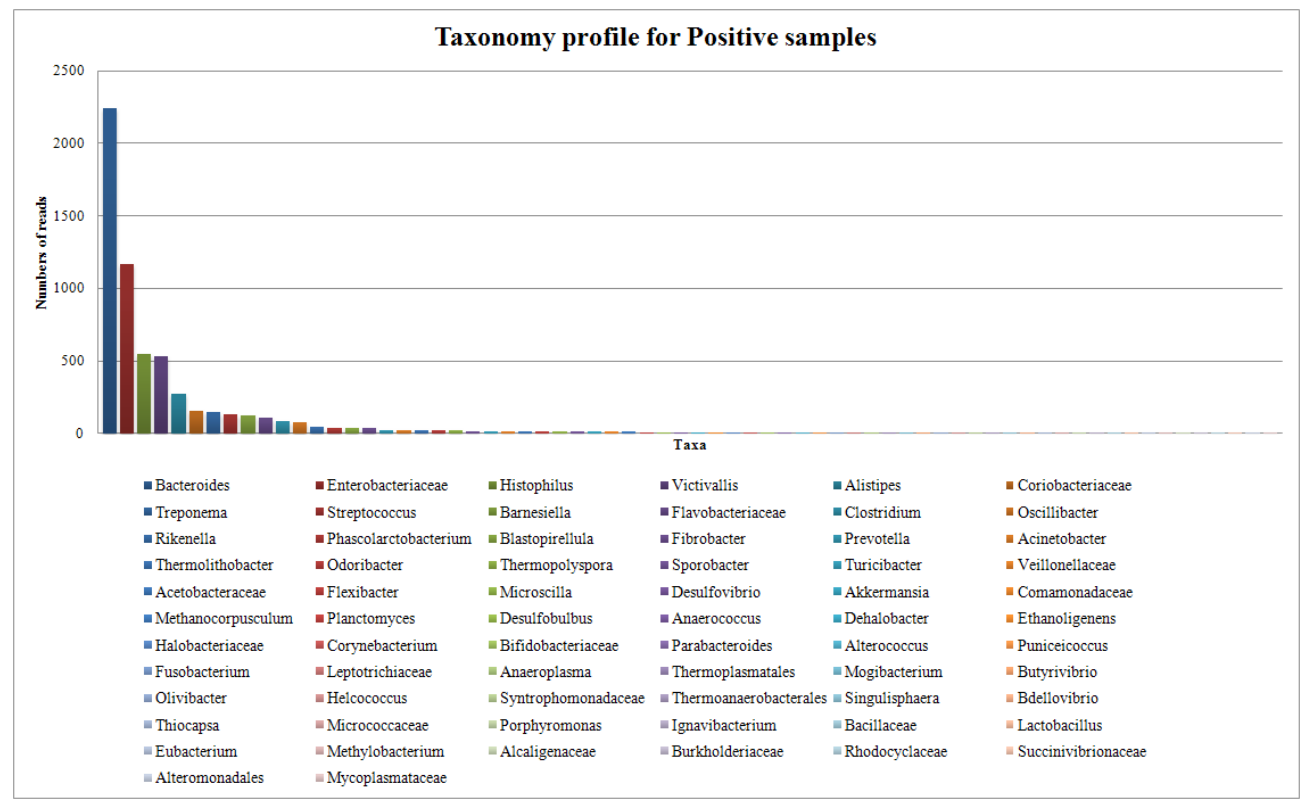

Figure 4. Taxonomic groups in the positive profile, generated in MEGAN, through BLASTn site, with $95 \%$ similarity. The taxa in legend are presented in descending order, from left to right. 
Reads evaluated in the control profile showed a diversal microbial attributes with the majority being negative Gram staining, no unknow, optimal growth temperature at $37^{\circ} \mathrm{C}$, oxygen requeriment facultative, host-associated habitat, pathogenic in humans, and rod-shaped (Figure 5).

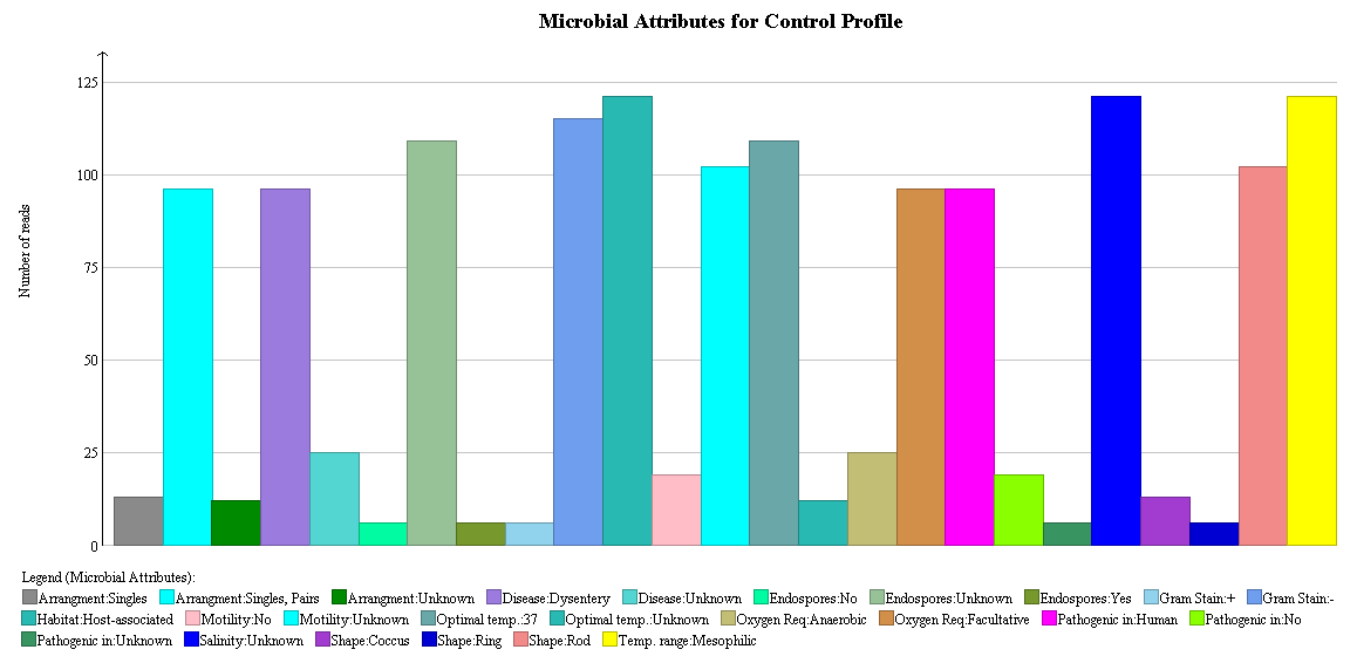

Figure 5. Attributes of the control profile. A balance can be observed in the morphological and physiological characteristics of the organisms in the community.

The positive profile differed from the control in several attributes (number of reads): Gram-stain; motility; optimal temperature; anaerobic facultative; habitat; disease; pathogenic; and shape (Figure 6). Phylograms with taxonomic classification of the OTUs is shown in Figure 7.

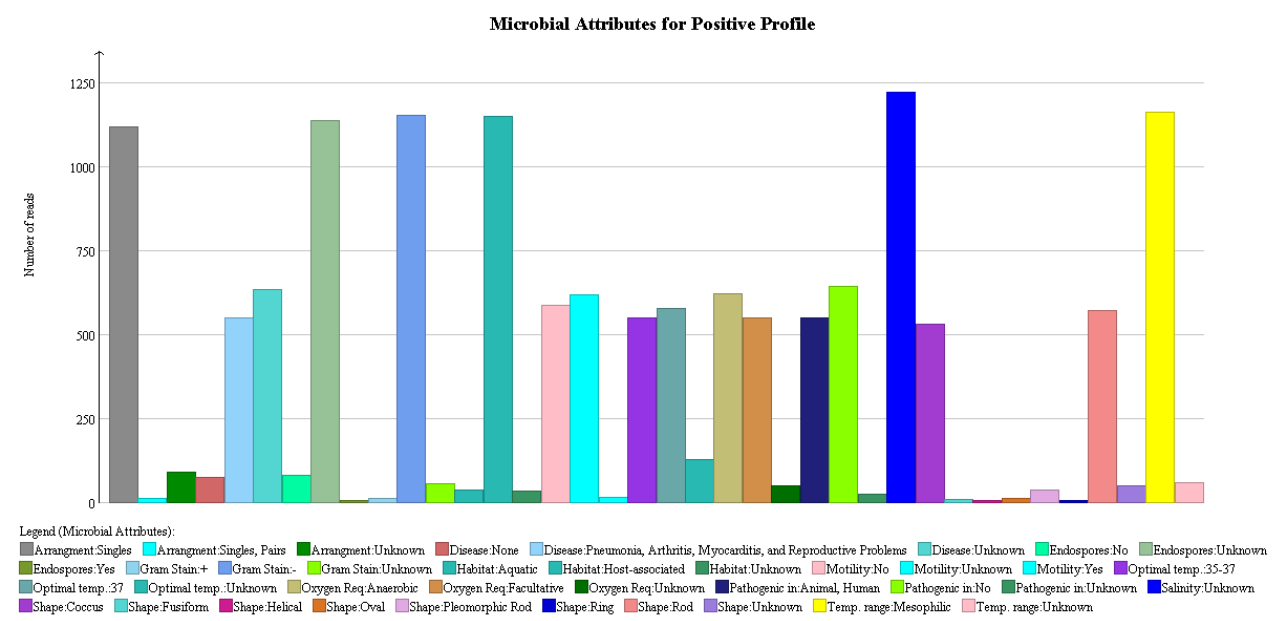

Figure 6. Microbial attributes of the positive profile. The presence of microorganisms was observed with attributes that were morphologically and physiologically distinct from those of the community control profile. 
$\mathbf{A}$

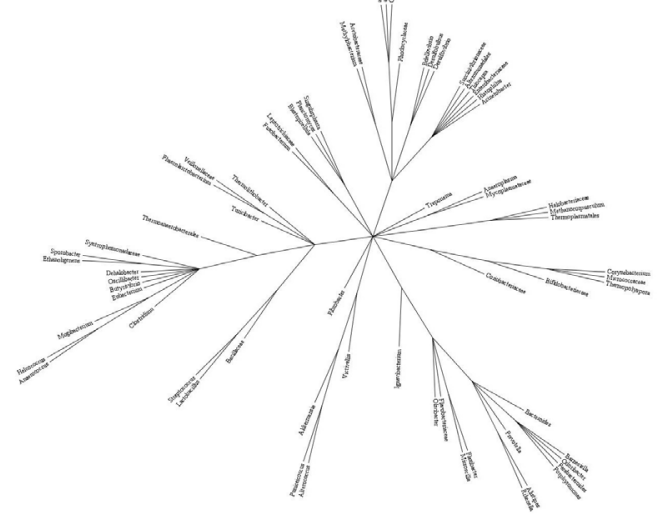

B

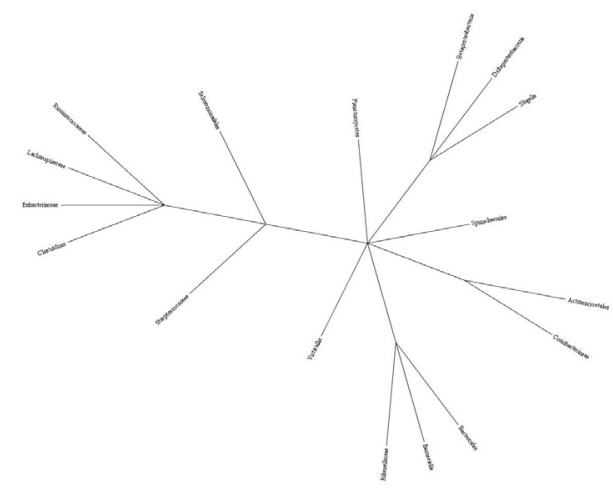

Figure 7. Phylograms of positive and control profiles. A. Positive profile. B. Control profile.

\section{DISCUSSION}

In this study, the microbial community composition profiles from vaginal mucosa samples from cows with genital disease or reproductive disorders (positive) and control animals showed significant differences, not only in the enrichment of taxa in the positive profile but also in the increased number of reads in this profile from the taxa that are part of the core of the bovine vaginal microbiota: Bacteroides, Enterobacteriaceae, Victivallis, Streptococcus, Selenomonadales, Treponema, Porphyromonadaceae, Alistipes, Clostridium, Coriobacteriaceae, Betaproteobacteria, Corynebacterineae, Cytophagaceae, and Oscillibacter, as compared to the control profile (Panangala et al., 1978; Otero et al., 2000). Specifically, in the positive profile, 53 additional taxa were found, as well as maintenance of organisms of the core microbiota, such as Bacteroides and Enterobacteriaceae, which have been described as part of the rumen flora in cattle (Wexler, 2007) as well as occasionally being the cause of disease (Dohmen et al., 1995; Kraipowich et al., 2000). One of the novel taxa, Histophilus can also be found in the genital tracts of cattle as opportunists, but can cause various disorders as well (van der Burgt et al., 2007); of all the pathogenic organisms, this genus has the largest representation, as well as correspondence to the clinical signs observed. Mycoplasmataceae also commonly inhabit mucous in cattle and can become pathogens (Nicholas et al., 2008), having a range of sites of infection greater than Histophilus; however, their representation was discreet in out sample, not corresponding to an infecting pathogen. The other taxa identified have not been described as pathogens in cattle.

Alterations in the composition of the microbial communities related to diseases in various organisms have been described, both through suppression (Ott et al., 2004; Manichanh et al., 2006) and colonization by new populations (Fredricks et al., 2005). Because of the high co-evolutionary association between individuals and microorganisms, a simple change in community composition can alter these interactions and trigger the change from a commensal to a pathogenic interaction. Changes in community composition, with concomitant, 
new emerging interactions, can arise from alterations in the abiotic factors in an ecosystem, which can open new ecological niches. The microbial attributes of both profiles evaluated in this study showed changes in the balance of the characteristics of the vaginal ecosystem between normal and disease states; for example, the increase of populations in the community of aerobic and facultative aerobic bacteria in the positive sample, such as Singulisphaera (Kulichevskaya et al., 2008), Olivibacter (Wang et al., 2008), and Comamonadaceae (Willems et al., 1991), from an almost totally anaerobic community in healthy animals. This change might be related to the formation of microecosystems; $\mathrm{pH}$ seems to change with the presence of taxa with representatives that live in acidic environments, such as Fibrobacter (Chow and Russell, 1992) and Lactobacillus, whose occurrence may result from the release of acidic compounds from the fermentation directed by these organisms and anaerobic organisms such as Victivalles (Zoetendal et al., 2003) and Bacteroides, all of which were identified in the positive sample profile. In addition, the availability of nutrients may be increased indirectly by organisms like Bdellovibrio, an intracellular parasite (Seidler and Starr, 1969), whose cycle may partially or completely involve lysing of their host, leading to release of ions and organic and inorganic compounds utilized in metabolic pathways of other bacteria. Akkermansia muciniphila might contribute to the emergent cycle of elements as well: through the fermentation of mucin, it has been shown to release sulfate in a free form, in addition to promoting a reduction in an organism's protection against pathogens, which is the function of the mucin protein (Derrien et al., 2004). Desulfovibrio could reduce the free sulfate (Voordouw, 1995), while Desulfobulbus could in turn oxidize the sulfide generated therefrom (Lien et al., 1998). Methanocorpusculum has been described as methanogenic using $\mathrm{H}_{2} / \mathrm{CO}_{2}$ or formate as substrates for methanogenesis, although some have also been shown to use alcohols as electron donors (Garcia et al., 2006). Similarly, Succinivibrionaceae has been described as capable of reducing methane (Stackebrandt and Hespell, 2006). These compounds could also be used by Histophilus in its methane, nitrogen, and sulfur metabolism, contributing to the context of infection. Furthermore, competition could occur between Histophilus, Acinetobacter, and Corynebacterium over the use of iron, because they have been shown to use iron in some metabolic processes and/or pathogens (Tremblay et al., 2006; Follmann et al., 2009; Zimbler et al., 2009).

In conclusion, the taxonomic profiles generated in this study showed that the composition of microbiota is consistent with vaginal health and disease status. Variations in microbiota might occur due to host-dependent and host-independent factors that were not considered here. However, the evidence of the relationship between reproductive disorders and changes in the composition of the vaginal microbiota of cattle appears to be multifactorial, and future studies may contribute to a greater understanding of this relationship. We propose that this knowledge may be used to modulate the vaginal microbiota for enhanced therapeutic efficacy in the treatment of these diseases.

\title{
ACKNOWLEDGMENTS
}

\author{
Research supported by CAPES.
}

\section{REFERENCES}

Baudoin E, Benizri E and Guckert A (2003). Impact of artificial root exudates on the bacterial community structure in bulk soil and maize rhizosphere. Soil Biol. Biochem. 35: 1183-1192. 
Brown CT, Davis-Richardson AG, Giongo A, Gano KA, et al. (2011). Gut microbiome metagenomics analysis suggests a functional model for the development of autoimmunity for type 1 diabetes. PLoS ONE 6: e25792.

Chow JM and Russell JB (1992). Effect of $\mathrm{pH}$ and monensin on glucose transport by Fibrobacter succinogenes, a cellulolytic ruminal bacterium. Appl. Environ. Microbiol. 58: 1115-1120.

Cole JR, Wang Q, Cardenas E, Fish J, et al. (2009). The Ribosomal Database Project: improved alignments and new tools for rRNA analysis. Nucleic Acids Res. 37: D141-D145.

Corbeil LB (2007). Histophilus somni host-parasite relationships. Anim. Health Res. Rev. 8: 151-160.

Derrien M, Vaughan EE, Plugge CM and de Vos WM (2004). Akkermansia muciniphila gen. nov., sp. nov., a human intestinal mucin-degrading bacterium. Int. J. Syst. Evol. Microbiol. 54: 1469-1476.

Dohmen MJW, Lohuis JACM, Huszenicza G, Nagy P, et al. (1995). The relationship between bacteriological and clinical findings in cows with subacute/chronic endometritis. Theriogenology 43: 1379-1388.

Follmann M, Ochrombel I, Kramer R, Trotschel C, et al. (2009). Functional genomics of pH homeostasis in Corynebacterium glutamicum revealed novel links between $\mathrm{pH}$ response, oxidative stress, iron homeostasis and methionine synthesis. BMC Genomics 10: 621 .

Fredricks DN, Fiedler TL and Marrazzo JM (2005). Molecular identification of bacteria associated with bacterial vaginosis. N. Engl. J. Med. 353: 1899-1911.

Garbeva P, van Veen JA and van Elsas JD (2004). Microbial diversity in soil: Selection of microbial populations by plant and soil type and implications for disease suppressiveness. Annu. Rev. Phytopathol. 42: 243-270.

Garcia J-L, Ollivier B and Whitman W (2006). The Order Methanomicrobiales. Prokaryotes 3: 208-230.

Huson D and Mitra S (2012). Introduction to the Analysis of Environmental Sequences: Metagenomics with MEGAN. Methods Mol. Biol. 856: 415-429.

Kent AD and Triplett EW (2002). Microbial communities and their interactions in soil and rhizosphere ecosystems. Annu. Rev. Microbiol. 56: 211-236.

Kraipowich NR, Morris DL, Thompson GL and Mason GL (2000). Bovine abortions associated with Bacteroides fragilis fetal infection. J. Vet. Diagn. Invest. 12: 369-371.

Kulichevskaya IS, Ivanova AO, Baulina OI, Bodelier PLE, et al. (2008). Singulisphaera acidiphila gen. nov., sp. nov., a non-filamentous, Isosphaera-like planctomycete from acidic northern wetlands. Int. J. Syst. Evol. Microbiol. 58: 1186-1193.

LeBlanc SJ (2008). Postpartum uterine disease and dairy herd reproductive performance: A review. Vet. J. 176: $102-114$.

Lien T, Madsen M, Steen IH and Gjerdevik K (1998). Desulfobulbus rhabdoformis sp. nov., a sulfate reducer from a water-oil separation system. Int. J. Syst. Bacteriol. 48: 469-474.

Lynch JM, Benedetti A, Insam H, Nuti MP, et al. (2004). Microbial diversity in soil: ecological theories, the contribution of molecular techniques and the impact of transgenic plants and transgenic microorganisms. Biol. Fertil. Soils 40: $363-385$.

Manichanh C, Rigottier-Gois L, Bonnaud E, Gloux K, et al. (2006). Reduced diversity of faecal microbiota in Crohn's disease revealed by a metagenomic approach. Gut 55: 205-211.

Nicholas R, Ayling RD and McAuliffe L (2008). Mycoplasma diseases of ruminants: disease, diagnosis and control. CAB International, Oxfordshire.

Otero C, Saavedra L, Silva de Ruiz C, Wilde O, et al. (2000). Vaginal bacterial microflora modifications during the growth of healthy cows. Lett. Appl. Microbiol. 31: 251-254.

Ott SJ, Musfeldt M, Wenderoth DF, Hampe J, et al. (2004). Reduction in diversity of the colonic mucosa associated bacterial microflora in patients with active inflammatory bowel disease. Gut 53: 685-693.

Panangala VS, Fish NA and Barnum DA (1978). Microflora of the cervico-vaginal mucus of repeat breeder cows. Can. Vet. J. 19: 83-89.

Pfützner H and Sachse K (1996). Mycoplasma bovis as an agent of mastitis, pneumonia, arthritis and genital disorders in cattle. Rev. Sci. Tech. 15: 1477-1494.

Schloss PD, Westcott SL, Ryabin T, Hall JR, et al. (2009). Introducing mothur: Open-source, platform-independent, communitysupported software for describing and comparing microbial communities. Appl. Environ. Microbiol. 75: 7537-7541.

Seidler RJ and Starr MP 1969. Factors affecting the intracellular parasitic growth of Bdellovibrio bacteriovorus developing within Escherichia coli. J. Bacteriol. 97: 912-923.

Seksik P, Rigottier-Gois L, Gramet G, Sutren M, et al. (2003). Alterations of the dominant faecal bacterial groups in patients with Crohn's disease of the colon. Gut 52: 237-242.

Singh J, Behal A, Singla N, Joshi A, et al. (2009). Metagenomics: Concept, methodology, ecological inference and recent advances. Biotechnol. J. 4: 480-494.

Stackebrandt E and Hespell R (2006). The Family Succinivibrionaceae. Prokaryotes 3: 419-429.

Tremblay YDN, Bahrami F and Niven DF (2006). Acquisition of haemoglobin-bound iron by Histophilus somni. Vet. Microbiol. 114: 104-114. 
Turnbaugh PJ and Gordon JI (2009). The core gut microbiome, energy balance and obesity. J. Physiol. 587: 4153-4158. van der Burgt G, Clark W and Knight R (2007). Cattle fertility problems and Histophilus somni. Vet. Rec. 160: 600.

Voordouw G (1995). The genus desulfovibrio: the centennial. Appl. Environ. Microbiol. 61: 2813-2819.

Wang L, Ten LN, Lee H-G, Im W-T, et al. (2008). Olivibacter soli sp. nov., Olivibacter ginsengisoli sp. nov. and Olivibacter terrae sp. nov., from soil of a ginseng field and compost in South Korea. Int. J. Syst. Evol. Microbiol. 58: 1123-1127.

Wexler HM (2007). Bacteroides: the Good, the Bad, and the Nitty-Gritty. Clin. Microbiol. Rev. 20: 593-621.

Willems A, De Ley J, Gillis M and Kersters K (1991). Comamonadaceae, a new family encompassing the Acidovorans rRNA complex, including Variovorax paradoxus gen. nov., comb. nov., for Alcaligenes paradoxus (Davis 1969). Int. J. Syst. Bacteriol. 41: 445-450.

Zimbler D, Penwell W, Gaddy J, Menke S, et al. (2009). Iron acquisition functions expressed by the human pathogen Acinetobacter baumannii. Biometals 22: 23-32.

Zoetendal EG, Plugge CM, Akkermans ADL and de Vos WM (2003). Victivallis vadensis gen. nov., sp. nov., a sugarfermenting anaerobe from human faeces. Int. J. Syst. Evol. Microbiol. 53: 211-215. 